\title{
Evidence-based cardiovascular disease prevention
}

\author{
Ferruccio De Lorenzo* \\ Consultant Physician in General Medicine \& Cardiovascular Disease Prevention, Hammersmith Hospital NHS Foundation Trust, London
}

In the next 20 years, more than $40 \%$ of the US population is expected to have some form of cardiovascular disease, and this will triple the total direct medical costs of caring for hypertension, coronary heart disease, heart failure, stroke, and other forms of cardiovascular disease from the current $\$ 273$ billion to more than $\$ 800$ billion, according to a new policy statement from the American Heart Association (AHA). In addition, the AHA estimates that the prevalence of cardiovascular disease will increase by approximately $10 \%$ over the next 20 years given no changes to prevention and treatment trends.

At present, cardiovascular disease is the leading cause of death in the US and accounts for $17 \%$ of overall healthcare expenditures. In the past, the medical costs of cardiovascular disease increased at an average annual rate of $6 \%$, and this growth in costs has been associated with an increase in life expectancy. That said, there are "many opportunities to further improve cardiovascular health while controlling costs," according to the AHA.

By 2030, the prevalence of cardiovascular disease is expected to increase $9.9 \%$, with the prevalence of heart failure and stroke increasing approximately $25 \%$. Total direct costs will increase to $\$ 818$ billion by 2030, according to the AHA estimates, and the total indirect cost to the US in terms of lost productivity is close to $\$ 275$ billion.

The total economic cost for cardiovascular disease per year to the European Union, including healthcare cost as well as cost for medications, informal care, plus lost opportunities, and productivity cost, is spiking at more than 196 billion Euro per year (Euro 196 billion is more than the annual budget of the EU).

Hypertension has the greatest projected medical cost, in part because of the aging population, although the increase in prevalence and cost is not explained fully by age. Rising obesity rates are also contributing to the increasing rates of hypertension. The AHA notes that the downstream medical costs of high blood pressure--including its impact on cardiovascular disease and stroke--approximately double the cost of hypertension, making it a "particularly valuable target to modify the future total costs of cardiovascular disease."

In the UK reductions in blood pressure and cholesterol concentrations accounted for just over half of the $34 \%$ fall in number of deaths from coronary heart disease from 2000 to 2007.

The EUROASPIRE III economic project emphasised the economic al and societal value of optimising cardiovascular disease prevention, with an average cost-effective ratio per quality-adjusted life (QUALY) years up to $£ 10,752$ in data from eight countries. Heads of States and Governments made commitments to the prevention and control of cardiovascular diseases in the political declaration from the UN Highlevel meeting on NCDs in September 2011. The main goal should be the identification of people at high risk of developing cardiovascular disease event through evidence-based preventative programmes.
The UK-National Screening Committee (UK-NSC), the British Medical Association (BMA) and the Academy of Medical Royal Colleges have recently expressed concern regarding the potential harms for healthcare (including cardiovascular) screening. In summary, the BMA and the Academy of Medical Royal Colleges issued a statement that there are significant risks with direct-to-consumer tests and many tests are unreliable and inaccurate. In addition, in their joint statement it was mentioned that is impossible for people to distinguish between private testing services that may do some good, and those of no value or even potentially harmful. The UK-NSC agrees that there are several accepted principles of screening that the NHS professionals should adhere to: only offer screening if there is proven evidence that, overall, the benefits of screening outweigh the potential harms. This means that screening should not be offered in the absence of known evidence. Surprisingly, healthcare companies, including the three top national UK players (BMI Healthcare, BUPA, Nuffield Healthcare), were found to offer screening tests with no proven benefits causing unnecessary worry to patients, resulting in higher insurance premiums, inadequate information, and increased number of referrals to NHS GPs. These conclusions were expressed by an expert panel (including members of the UK-NSC) that assessed the findings and reports of screening programmes offered by these companies.

The UK health screening market was assessed to be worth around $£ 100$ million in 2009 (data from Laing \& Buisson). It is quite clear, therefore, that there is a consolidated interest in protecting the "status quo" of the screening industry. In addition, in the UK fewer than half the patients who are started on a statin therapy by their general practitioner are at high risk of developing heart disease and eligible for treatments under current guidelines (study funded by the National Institute for Health Research, and carried out by researchers from the University of Birmingham). Therefore, over a two-year period only about one in three patients who should be taking statins had prescribed them but meanwhile the drugs were prescribed inappropriately to one in 10 low risk patients. This means that of those who were prescribed a statin, only 42\% (21 101 of 50558 ) were considered to need one.

Treating people at high risk of cardiovascular disease with aspirin, a statin, and two blood pressure lowering drugs is highly cost effective and would save about as many premature deaths as the population strategies. Setting up more cardiovascular evidence based screening services or centres throughout the countries should be seriously

Correspondence to: Ferruccio De Lorenzo, Honorary Consultant Physician in General Medicine and Cardiovascular Disease Prevention, Hammersmith Hospital NHS Foundation Trust, Imperial College, Du Cane Road, London, UK, E-mail: fdlx@btinternet.com

Received: January 09, 2017; Accepted: January 25, 2017; Published: January 27, 2017 
considered. The major investment would not be in the equipment, which is now relatively inexpensive and portable, but in trained staff. Asymptomatic individuals should be referred to such centres by their own physicians. Cardiovascular disease is largely preventable through training an adequate workforce, using clinical practice guidelines to improve care, improving management of population risk factors.

Copyright: $@ 2017$ Lorenzo FD. This is an open-access article distributed under the terms of the Creative Commons Attribution License, which permits unrestricted use, distribution, and reproduction in any medium, provided the original author and source are credited. 